Bundesgesundheitsbl 2014 · 57:1331-1337 DOI 10.1007/s00103-014-2053-x

Online publiziert: 30 . September 2014

(c) Springer-Verlag Berlin Heidelberg 2014

I.-K. Wolf · M. Busch · M. Lange - P. Kamtsiuris - R. Doelle · A. Richter · R. Kuhnert • T. Ziese $\cdot$ H. Knopf $\cdot$ C. Scheidt-Nave

Robert Koch-Institut, Berlin, Deutschland

\title{
Mortalitäts-Follow-up der Studie zur Gesundheit Erwachsener in Deutschland (DEGS)
}

\section{Methodik und erste Ergebnisse}

\section{Hintergrund und Problemstellung}

Das Mortalitäts-Follow-up von Teilnehmerinnen und Teilnehmern der bundesweiten Studie zur Gesundheit Erwachsener in Deutschland (DEGS) eröffnet die Perspektive, im Rahmen des kontinuierlichen bundesweiten Gesundheitsmonitorings Unterschiede in der Mortalität bestimmter Bevölkerungsgruppen (z. B. zwischen Diabetikern und Nichtdiabetikern oder auch nach sozialer Lage) zu beobachten. Darüber hinaus lassen sich Fragestellungen zum Einfluss von Versorgungsqualität und zum Stellenwert gesundheitlicher, sozialer und funktioneller Einschränkungen, bezogen auf Krankheitsentwicklung und Krankheitsverläufe, beantworten. Im Rahmen der ersten Welle der Studie zur Gesundheit Erwachsener in Deutschland (DEGS1) [1-4] wurden die Studienteilnehmerinnen und -teilnehmer des Bundesgesundheitssurveys 1998 (BGS98) [5] erneut zur Studienteilnahme eingeladen. Für die bereits Verstorbenen der BGS98-Kohorte sollten die Todesursachen erhoben werden.

Da in der Bundesrepublik Deutschland kein nationales Mortalitätsregister existiert [6] und diesbezüglich länderspezifische Unterschiede hinsichtlich der gesetzlichen Regelungen und Zuständigkeiten bestehen [7-9], hat das Robert Koch-Institut (RKI) im Jahr 2011 eine Bestandsaufnahme zu den Rahmenbedingungen durchgeführt [10], um anschließend (2012) die Todesursachen der Verstorbenen aus der BGS98Kohorte zu erheben. In allen 16 Bundes- ländern wurden die Abläufe zur Todesursachenrecherche anhand einer Stichprobe getestet. Durchführung und Ergebnisse dieser Machbarkeitsstudie wurden bereits publiziert [10]. Auf Basis der erprobten Abläufe wurde eine Vervollständigung der Todesursachenrecherche angestrebt.

Nach aktuellen Bestimmungen im Bundesdatenschutzgesetz [9] und in den Datenschutzgesetzen der Länder [8] ist zu Lebzeiten von den Studienteilnehmerinnen und -teilnehmern eine schriftliche Einwilligungserklärung zur Einsicht in die Todesbescheinigung einzuholen. Seit Sommer 2010 wurde das Einholen der Einwilligungserklärung zur Todesursachenrecherche in DEGS integriert. Für bereits Verstorbene konnte dies jedoch nicht mehr nachgeholt werden. Grundsätzlich ist es aber möglich, Recherchen zu den Todesursachen auch ohne vorab eingeholte Einwilligungserklärung durchzuführen, wenn die Einholung nicht mehr möglich ist, der Forschungszweck einem legitimierten Gemeinschaftsinteresse dient und die erforderlichen Datenschutzbedingungen eingehalten werden [11]. Die Datenerhebung setzt jedoch immer befürwortende Stellungnahmen des Bundes- und der Landesdatenschutzbeauftragten voraus. Die datenschutzrechtlichen Bedingungen, Zugangswege und Verfahrensweisen in den Ländern wurden bereits dargestellt [10].

\section{Datenbasis und Methodik}

Grundgesamtheit der Studie waren die Teilnehmerinnen und Teilnehmer des
BGS98 $(\mathrm{N}=7124)$. Ausgeschlossen wurden 145 Personen, die keinen weiteren Kontakt wünschten. Somit standen 6979 Personen der BGS98-Kohorte $(98,0 \%)$ für das Mortalitäts-Follow-up zur Verfügung.

Im Rahmen von DEGS1 wurden diese Personen zur erneuten Befragung und Untersuchung eingeladen. Hierzu wurden diese sequenziell entsprechend der Routenplanung der Studie für die 180 Untersuchungsorte zunächst wiederholt angeschrieben und ggf. telefonisch oder persönlich am Wohnort kontaktiert $[2-4,12]$. Die Kontaktaufnahme fand etwa 5 Wochen vor der Anreise des Feldteams am jeweiligen Sample Point (also von Oktober 2008 bis Oktober 2011) statt. Vor der Einladung wurde die Aktualität der Anschriften über die Einwohnermeldeämter (EMA) recherchiert (zuletzt September 2011). Dabei wurde auch die Information erhoben, ob eine Person verstorben war [12]. Um Todesursachen über die Datenhalter (überwiegend die Gesundheitsämter) recherchieren zu können, müssen neben Namen, letzter Adresse und Geburtsdatum auch das Sterbedatum und der Sterbeort bekannt sein. Allgemein zugängliche Daten, wie z. B. eine neue Adresse bei verzogenen Personen, können mit einer einfachen Melderegisterauskunft ermittelt werden. Um Sterbedatum und Sterbeort zu ermitteln, bedarf es einer erweiterten Abfrage, bei der ein berechtigtes Interesse nachgewiesen werden muss. Die Datenhalter der Todesbescheinigungen (überwiegend die Gesundheitsämter, s. • Tab. 2) 


\begin{tabular}{|lll}
\hline Tab. 1 & Hauptdiagnosen als Todesursachen gemäß Todesbescheinigung & Zeitdauer von Beginn der \\
\hline Felder & Krankheiten & $\begin{array}{l}\text { ICD-Kodierung } \\
\text { Krankheit bis zum Tod }\end{array}$ \\
\hline $1 \mathrm{a}$ & $\begin{array}{l}\text { Unmittelbar zum Tode führende } \\
\text { Krankheit }\end{array}$ \\
\hline 1b & Vorangegangene Ursachen & \\
\hline $1 \mathrm{c}$ & Grundleiden & \\
\hline 2 & Andere wesentliche Krankheiten & \\
\hline Epikrise & Weitere Angaben \\
\hline
\end{tabular}

fordern für die Todesursachenrecherche neben den oben beschriebenen Angaben zur Person der Verstorbenen eine befürwortende Stellungnahme des Landesdatenschutzbeauftragten und ggf. anderer Entscheidungsträger (z. B. des Landesgesundheitsministeriums). Weitere Details über die Zugangsbedingungen in den einzelnen Bundesländern wurden an anderer Stelle publiziert [10].

In Deutschland sind die Formulare zur Ausstellung von Todesbescheinigungen in den Bundesländern - und zum Teil selbst innerhalb einzelner Bundesländer - inhaltlich und formal unterschiedlich gestaltet. Die WHO empfiehlt internationale Kriterien zur inhaltlichen Gestaltung der Todesbescheinigungen [13, 14]. Diese Empfehlungen beziehen sich auf den Teil der Todesbescheinigung, in dem die „Hauptdiagnosen“ (s. - Tab. 1: unmittelbar zum Tode führende Krankheit, vorangegangene Ursachen, Grundleiden, andere wesentliche Krankheiten und Epikrise) einzutragen sind und der von den einzelnen Bundesländern weitestgehend übernommen wird. Nach WHO-Vorgaben ist das Grundleiden (s. - Tab. 1, Feld 1c) entscheidend für die Zuordnung der Todesursache. Die zusätzlichen Informationen, die neben dem von den Ärzten eingetragenen Grundleiden erhoben werden, können zur Plausibilitätsprüfung herangezogen werden.

Die für die Todesursachenrecherche erhobenen Daten wurden daher nach folgenden Kriterien für die Auswertung ausgewählt:

- Einheitliche Erfassung in den Bundesländern,

- Übereinstimmung mit internationalen Empfehlungen zur Erfassung von Todesursachen der WHO [13],

- Möglichkeit einer multikausalen Todesursachenerhebung.
Für das Mortalitäts-Follow-up wurden nur die in allen Bundesländern weitgehend einheitlich erfassten Angaben zu den Hauptdiagnosen als Todesursachen erhoben. In • Tab. 1 sind die ausgewählten Informationen, entsprechend ihrer Anordnung auf den Todesbescheinigungen, dargestellt.

Dabei ist zu bemerken, dass das letzte Feld, „Epikrise“, international in Todesbescheinigungen nicht üblich ist. Da es aber in Deutschland in den meisten Todesbescheinigungen vorhanden ist und einige Ärzte erst im Feld „Epikrise“ die wichtigsten Informationen zu den Todesursachen eintragen, wurde es für die Todesursachenrecherche berücksichtigt.

Nach Empfehlungen der WHO sollen für jedes Feld auf der Todesbescheinigung die entsprechende Krankheit und die Zeitdauer vom Beginn der Krankheit bis zum Eintritt des Todes von den Leichenschauärzten eingetragen werden. In Deutschland sind die Todesbescheinigungen in vielen Bundesländern zusätzlich mit einer Spalte für die ICD-Kodierung ausgestattet. In einigen Bundesländern wird die ICD-Kodierung jedoch erst im Statistischen Landesamt vorgenommen. Die in - Tab. 1 dargestellten Informationen aus den Leichenschauscheinen wurden in die Dokumentation der Todesursachen übernommen. Lagen $\mathrm{Ob}-$ duktionsdiagnosen vor, so wurden diese statt der Diagnosen des Leichenschauscheins berücksichtigt. Nach Erhebung und Überprüfung der multikausalen Todesursachen wurden alle Diagnosen anonymisiert an das Deutsche Institut für Medizinische Dokumentation und Information (DIMDI) übermittelt. Dort erfolgte eine Zuordnung zu ICD-Codes mittels des international vergleichbaren IRIS-Systems $[15,16]$. Die eigentliche Todesursache wurde anhand der Hauptdiagnosen aus der Todesbescheinigung bestimmt.
Dabei wurden sämtliche eingetragenen Diagnosen und die Angaben zum Zeitraum von Beginn der Erkrankung bis zum Tod berücksichtigt. Nach Rückübermittlung der Todesursachen durch das DIMDI an das RKI wurden die am RKI vorhandenen Personendaten für alle recherchierten Fälle gelöscht. Für die statistische Analyse in unserer Studie wurden die Todesursachen (Grundleiden) in die Gruppen Herz-Kreislauf-Erkrankungen (ICD10: I00-I99), Krebserkrankungen (ICD-10: C00-C97), keine eindeutig zu ermittelnde Ursache (ICD-10: R95-R99) und andere bekannte Ursache (alle anderen ICD-10Kodierungen) zusammengefasst.

Als Überlebenszeit wurde die Zeit in Tagen von der Basisuntersuchung im Rahmen des BGS98 bis zum Sterbedatum bei Verstorbenen oder bis zum Zeitpunkt des letzten Kontaktes bei Überlebenden berechnet. Überlebende Personen wurden zum Zeitpunkt des letzten Kontaktes zensiert. Bei BGS98-Teilnehmern, die im Rahmen von DEGS1 im Studienzentrum nachuntersucht wurden, wurde hierzu der DEGS1-Untersuchungszeitpunkt gewertet. Bei DEGS1-Teilnehmern, die nur telefonisch oder schriftlich wieder befragt wurden, wurde das Datum des Telefoninterviews bzw. das Rücksendedatum des Fragebogens herangezogen. Bei DEGS1Nichtteilnehmern wurde das Datum der letzten EMA-Abfrage gewertet.

Sterberaten wurden pro 1000 Personen pro Jahr insgesamt und getrennt nach Geschlecht berechnet. Anhand von Cox-Regressionsmodellen wurde die Überlebenswahrscheinlichkeit nach Alter und nach Geschlecht geschätzt. Das hierauf beruhende Sterberisiko wurde als Hazard Ratio (HR) mit $95 \%$-Konfidenzintervallen (KI) berechnet. Unterschiede in der mittleren Lebenserwartung zwischen Frauen und Männern im Altersspektrum 18 bis 79 Jahre wurden anhand marginaler Effekte der Cox Regressionsmodelle quantifiziert.

\section{Ergebnisse}

Bis einschließlich September 2011 waren insgesamt 671 Personen (285 Frauen, 386 Männer) verstorben. In allen Bundesländern und somit für alle 671 Verstorbenen konnten das Sterbedatum und 
Bundesgesundheitsbl 2014 · 8:1331-1337 DOI 10.1007/s00103-014-2053-x

(c) Springer-Verlag Berlin Heidelberg 2014

\section{I.-K. Wolf · M. Busch · M. Lange · P. Kamtsiuris · R. Doelle · A. Richter · R. Kuhnert · T. Ziese · H. Knopf · C. Scheidt-Nave Mortalitäts-Follow-up der Studie zur Gesundheit Erwachsener in Deutschland (DEGS). Methodik und erste Ergebnisse}

\section{Zusammenfassung}

Ziele. Im Rahmen der Studie zur Gesundheit Erwachsener in Deutschland (DEGS) hat das Robert Koch-Institut (RKI) ein bundesweites Mortalitäts-Follow-up durchgeführt. Da in Deutschland kein nationales Mortalitätsregister existiert, wurden Todesfälle und Todesursachen individuell und unter Berücksichtigung von länderspezifischen datenschutzrechtlichen Rahmenbedingungen recherchiert.

Methodik. Als Datenbasis diente der Bundes-Gesundheitssurvey 1998 (BGS98) mit 7124 Studienteilnehmerinnen und Teilnehmer im Alter von 18 bis 79 Jahren. Im Rahmen der ersten Datenerhebungswelle von DEGS (DEGS1) wurden 6979 BGS98-Teilnehmende (98\%), die einer weiteren Kontaktaufnahme zugestimmt hatten, zwischen Oktober 2008 und Oktober 2011 zur erneuten Studienteilnahme eingeladen. In diesem Zusammenhang wurden Vitalstatus und für bereits verstorbene Studienteilnehmer die Todesursachen ermittelt. Überlebenswahrscheinlichkeiten und Sterberaten wurden nach Alter und Geschlecht berechnet und die häufigsten Todesursachen in ICD10-Krankheitsgruppen zusammengefasst analysiert.

Ergebnisse. Insgesamt waren 671 Personen (285 Frauen, 386 Männer) im Zeitraum zwischen den beiden Surveykontakten verstorben. Für alle Verstorbenen konnte das Sterbedatum und für 539 (80,3\%) konnten die Todesursachen recherchiert werden. Bei einer medianen Nachbeobachtungszeit von 12,0 Jahren standen für die Überlebenszeitanalyse $80.742,5$ Personenjahre zur Verfügung. Die rohe Sterberate betrug insgesamt 8,3 pro 1000 Personen/Jahr (Frauen: 7,2; Männer: 9,5). Unter 539 Todesfällen mit Information zur Todesursache waren 209 (38,8\%) auf eine Herz-Kreislauf-Erkrankung, 188 (34,9\%) auf eine Krebserkrankung,
$135(25,0 \%)$ auf eine andere Ursache und 7 $(1,3 \%)$ auf keine eindeutige Ursache zurückzuführen.

Fazit. Im Rahmen des bundesweiten Gesundheitsmonitorings des RKI konnte ein Mortalitäts-Follow-up in die längsschnittliche Komponente von DEGS integriert werden. Sterberate und ursachenspezifische Mortalität im Zusammenhang mit weit verbreiteten chronischen Erkrankungen und Risikofaktoren liefern wichtige ergänzende Informationen für Einschätzungen zu Präventionspotenzial und gesundheitlicher Versorgungsqualität bei Erwachsenen in Deutschland. Voraussetzung hierfür sind regelmäßige und vollständige Todesursachenrecherchen.

\section{Schlüsselwörter}

Mortalitäts-Follow-up .

Todesursachenrecherche $\cdot$ Bundesweites Gesundheitsmonitoring · DEGS · Deutschland

\section{Mortality follow-up of the German Health Interview and Examination Survey for Adults (DEGS). Methods and first results}

\section{Abstract}

Objectives. Within the framework of the German Health Interview and Examination Survey for Adults (DEGS), the Robert Koch Institute (RKI) conducted a nationwide mortality follow-up study. As there is no national mortality register in Germany, mortality and causes of death were investigated individually and under observance of state-specific data protection conditions.

Methods. The German Health Interview and Examination Survey 1998 (GNHIES98) provided the database including 7,124 participants aged 18-79 years. A total of 6,979 participants of GNHIES98 (98\%) who consented to be re-contacted were invited between October 2008 and October 2011 to also participate in the first data collection wave of DEGS (DEGS1). In this context, the vital status and the causes of death for deceased participants were assessed. Age- and sex-specific probabilities of survival and death rates were calculated and grouped by main causes of death according to ICD-10 groups.

Results. A total of 671 individuals ( 285 women, 386 men) died between the two survey contacts. For all deceased persons the date of death and for 539 (80.3\%) the causes of death could be determined. With a median follow-up time of 12.0 years, $8,0742.5$ person years were available for survival analysis. The crude overall death rate amounted to 8.3 per 1,000 persons-years (women: 7.2; men: 9.5$)$. Among 539 persons with available information on causes of death, 209 (38.8\%) were attributable to cardiovascular diseases, $188(34.9 \%)$ to cancer, $135(25.0 \%)$ to other causes, and seven (1.3\%) could not be unambiguously assigned.

Conclusions. A mortality follow-up was successfully integrated in the longitudinal component of DEGS as part of the national health monitoring at the RKI. Death rates and causespecific mortality in relation to highly prevalent chronic diseases and risk factors provide essential information for assessing the potential of prevention and quality of care among adults in Germany. This requires a regular and complete conduction of mortality follow-ups.

Keywords

Mortality follow-up - Cause of death research . National health monitoring · DEGS . Germany der Sterbeort ermittelt werden. In 12 von 16 Bundesländern konnten zudem für alle Verstorbenen die Todesursachen ermittelt werden. Der Erfolg und weitere Charakteristika der Todesursachenrecherche nach Bundesland sind in $\bullet$ Tab. 2 dargestellt. Nordrhein-Westfalen (NRW) ist das einzige Bundesland, in dem aufgrund landes- spezifischer Datenschutzregelungen generell keine Todesursachenrecherche ohne Einwilligungserklärung und zusätzlicher Entbindung von der ärztlichen Schweigepflicht durchgeführt werden konnte [10]. Auf NRW entfielen 107 Todesfälle, 16,0\% aller Todesfälle.
Weitere Datenverluste waren bedingt durch Verjährung der Aufbewahrungsfrist in Rheinland-Pfalz $(n=5)$ sowie durch kurze Aufbewahrungsfristen und schwierige Zugangsbedingungen in Ber$\operatorname{lin}(\mathrm{n}=11)$.

In Brandenburg konnte in einem Fall keine Todesursache erhoben werden, da 


\section{Aus den Herausgeberinstituten}

Tab. 2 Ergebnisse der Todesursachenrecherche nach Bundesländern und Aufbewahrungsorten der Todesbescheinigungen

\begin{tabular}{|c|c|c|c|c|c|}
\hline Bundesland & Aufbewahrungsort der TBs & $\begin{array}{l}\text { Anzahl Ver- } \\
\text { storbener }^{\mathrm{a}}\end{array}$ & $\begin{array}{l}\text { TU } \\
\text { recherchiert }\end{array}$ & $\begin{array}{l}\text { TU } \\
\text { ungeklärt }\end{array}$ & Gründe für ungeklärte TU \\
\hline Baden-Württemberg & GA des Sterbeortes & 52 & 52 & 0 & 0 \\
\hline Bayern & GA des Sterbeortes & 95 & 95 & 0 & 0 \\
\hline Berlin & $\begin{array}{l}\text { Statistisches Landesamt, TB dort nur } \\
\text { durch Leichenschauärzte einsehbar }\end{array}$ & 26 & 15 & 11 & $\begin{array}{l}11 \text { Fälle: TBs aufgrund der kurzen } \\
\text { Aufbewahrungsfristen nicht mehr } \\
\text { vorhanden, bzw. Recherchen über } \\
\text { Leichenschauärzte nicht erfolgreich }\end{array}$ \\
\hline Brandenburg & GA des Sterbeortes & 32 & 31 & 1 & $\begin{array}{l}1 \text { Fall: Letzter Wohnort, aber nicht } \\
\text { Sterbeort war Brandenburg. Die TB } \\
\text { war nicht aufzufinden }\end{array}$ \\
\hline Bremen & $\begin{array}{l}\text { Bremen: Institut für Rechtsmedizin } \\
\text { Bremerhaven: Magistrat der Stadtgemein- } \\
\text { de Bremerhaven } \\
\text { Alle: Bremer Mortalitätsindex }\end{array}$ & 6 & 6 & 0 & 0 \\
\hline Hamburg & GA Wandsbek & 7 & 7 & 0 & 0 \\
\hline Hessen & GA des Sterbeortes & 43 & 43 & 0 & 0 \\
\hline $\begin{array}{l}\text { Mecklenburg- } \\
\text { Vorpommern }\end{array}$ & GA des Sterbeortes & 27 & 27 & 0 & 0 \\
\hline Niedersachsen & GA des Sterbeortes & 37 & 36 & 1 & $\begin{array}{l}1 \text { Fall: Person zunächst nicht auffind- } \\
\text { bar, Sterbedatum und Sterbedatum } \\
\text { konnten erst nach Abschluss der } \\
\text { Datenerhebung ermittelt werden }\end{array}$ \\
\hline Nordrhein-Westfalen & GA des Sterbeortes & 107 & 0 & 107 & $\begin{array}{l}107 \text { Fälle: keine Zustimmung des } \\
\text { Datenschutzbeauftragten, da Recher- } \\
\text { che nur mit Einverständniserklärung } \\
\text { und ärztlicher Schweigepflichtent- } \\
\text { bindung möglichwar }\end{array}$ \\
\hline Rheinland-Pfalz & GA des Sterbeortes & 26 & 21 & 5 & $\begin{array}{l}5 \text { Fälle: TBs aufgrund der kurzen } \\
\text { Aufbewahrungszeiten nicht mehr } \\
\text { vorhanden }\end{array}$ \\
\hline Saarland & GA des Sterbeortes & 17 & 17 & 0 & 0 \\
\hline Sachsen & GA des Sterbeortes & 79 & 79 & 0 & 0 \\
\hline Sachsen-Anhalt & $\begin{array}{l}\text { Bis 01.03.2005 GA des Wohnortes, danach } \\
\text { GA des Sterbeortes }\end{array}$ & 49 & 49 & 0 & 0 \\
\hline Schleswig-Holstein & GA des Wohnortes & 13 & 13 & 0 & 0 \\
\hline Thüringen & GA des Wohnortes & 48 & 48 & 0 & 0 \\
\hline Bundesweit & & 7 & 0 & 7 & $\begin{array}{l}7 \text { Fälle: Probanden im Ausland ver- } \\
\text { storben }\end{array}$ \\
\hline Gesamt & & 671 & 539 & 132 & \\
\hline
\end{tabular}

GA Gesundheitsamt, TBTodesbescheinigung, TUTodesursache.

alm Datensatz des Bundes-Gesundheitssurveys 1998 (BGS98-Datensatz) kann die Anzahl der Verstorbenen in den Bundesländern von Angaben zur Anzahl der Verstorbenen nach Bundesland in - Tab. 2 abweichen, da einige Probanden im Zeitraum zwischen der BGS98-Erhebung und dem Mortalitäts-Follow-up verzogen oder in anderen Bundesländern verstorben waren und dort recherchiert wurden. Im BGS98-Datensatz sind die Verstorbenen aber generell dem ersten dokumentierten Wohnort zugeordnet

die Todesbescheinigung nicht mehr auffindbar war. Die verstorbene Person war mit letztem Wohnsitz in Brandenburg gemeldet, aber nicht dort verstorben. Im zuständigen Gesundheitsamt in Brandenburg werden nur Todesbescheinigungen für Personen aufbewahrt, die in Brandenburg versterben. Da in dem Bundesland, in dem die Person verstorben war, für die Aufbewahrung der letzte Wohnort zugrunde lag, konnte im konkreten Fall keine Todesbescheinigung aufgefun- den werden. (s. - Tab. 2). In Niedersachsen konnte für einen Fall keine Todesursache recherchiert werden, da der Vitalstatus zunächst nicht zu klären war sowie Sterbedatum und Sterbeort erst nach Abschluss der Datenerhebung bekannt wurden. Auch die Todesursachen von im Ausland verstorbenen Kohortenmitgliedern $(\mathrm{n}=7)$ waren nicht mehr recherchierbar (- Tab. 2).

Unter 539 Todesfällen (80,3\%), für die eine Todesursache recherchiert werden konnte, war das Grundleiden bei 209 Personen $(38,8 \%)$ einer Herz-Kreislauf-Erkrankung (ICD-10: I00-I99), bei 188 Personen $(34,9 \%)$ einer Krebserkrankung (C00-C97) und bei 135 Personen (25,0\%) einer anderen Ursache zuzuordnen. In 7 Fällen (1,3\%) konnte keine eindeutige Ursache (R95-R99) ermittelt werden.

Die rohe Sterberate im Beobachtungszeitraum betrug insgesamt 8,3 pro 1000 Personen pro Jahr und lag bei Männern mit 9,5 pro 1000 pro Jahr insge- 

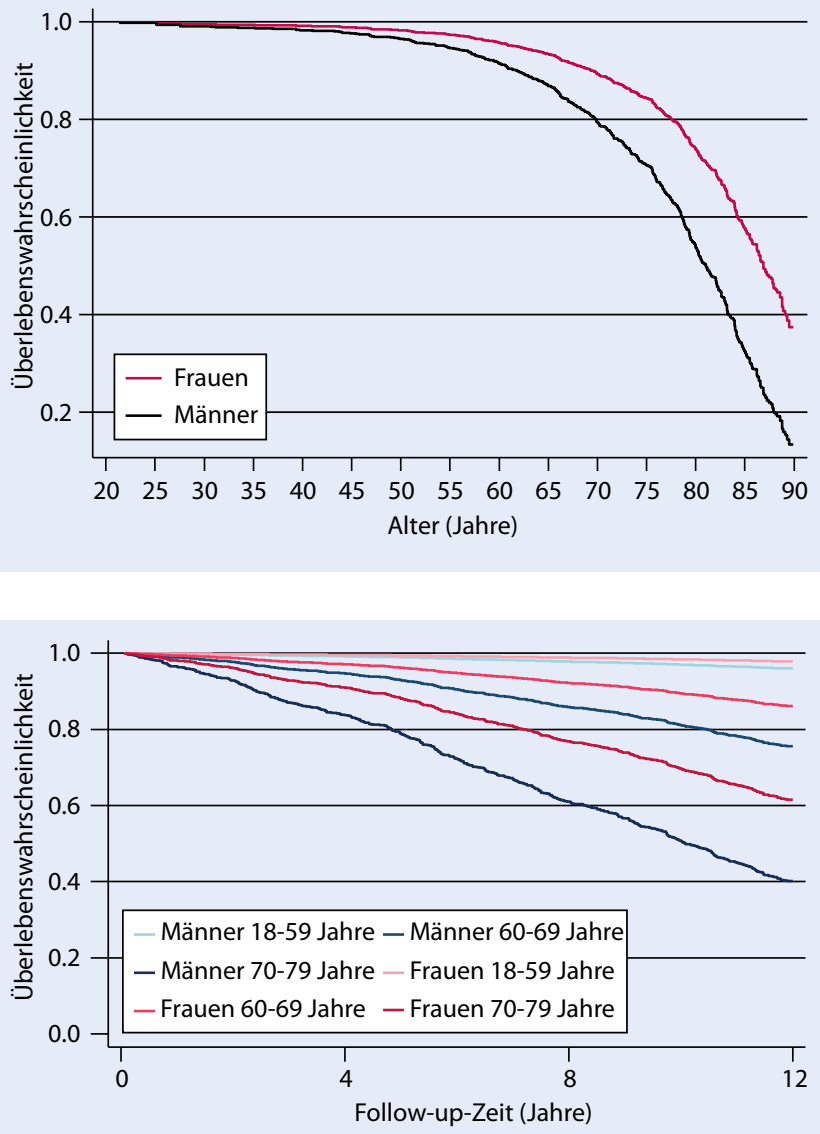

Abb. $1<$ Überlebenswahrscheinlichkeit von Teilnehmenden des Bundes-Gesundheitssurveys 1998 (BGS98, $\mathrm{n}=6979$ ) im Altersverlauf nach Geschlecht (Schätzungen mit CoxRegression)

Abb. $2<$ Überlebenswahrscheinlichkeit von Teilnehmenden des Bundes-Gesundheitssurveys 1998 (BGS98, $\mathrm{n}=6979$ ) während des Follow-ups nach Altersgruppen und Geschlecht (Schätzungen mit Cox-Regression) samt signifikant höher als bei Frauen mit 7,2 pro 1000 pro Jahr $(\mathrm{p}=0,0002)$. Frauen hatten insgesamt eine höhere Überlebenswahrscheinlichkeit als Männer (• Abb. 1). In der für Altersunterschiede korrigierten Analyse hatten Männer dabei ein insgesamt etwa doppelt so hohes Sterberisiko wie Frauen (HR: 2,1; $95 \%$-KI: 1,77-2,38). Der Unterschied in der mittleren Lebenserwartung zwischen Frauen und Männern im Altersspektrum 18 bis 79 Jahre betrug insgesamt 6,4 Jahre. In einer altersgruppenspezifischen Analyse zeigte sich, dass der Geschlechtsunterschied in der Überlebenswahrscheinlichkeit in allen Altersgruppen bestand und sich mit zunehmendem Lebensalter vergrößerte (• Abb. 1 und 2).

\section{Diskussion}

Im Rahmen des bundesweiten Gesundheitsmonitorings konnten in der Studie zur Gesundheit Erwachsener in Deutschland (DEGS) Abläufe zur Erhebung von Todesursachen verstorbener Studienteilnehmerinnen und -teilnehmer recher- chiert und etabliert werden. Die Basis bildete hierbei die Studienpopulation des BGS98, die im Rahmen der durchschnittlich 12 Jahre später durchgeführten Erhebungswelle 2008 bis 2011 (DEGS1) wieder kontaktiert wurde, sofern die Teilnehmenden ihre Einwilligung hierzu gegeben hatten. Da kein nationales Mortalitätsregister existiert, war eine getrennte Todesursachenrecherche in allen 16 Bundesländern notwendig. $\mathrm{Zu}$ beachten ist, dass aktuelle Datenschutzbestimmungen die zu Lebzeiten eingeholte schriftliche Einwilligung der Studienteilnehmerinnen und -teilnehmer voraussetzen, um Informationen zur Todesursache bei Datenhaltern (in der Regel die regionalen Gesundheitsämter) zu erhalten. Für wissenschaftliche Untersuchungen von Interesse für die Allgemeinheit kann jedoch in fast allen Bundesländern (außer in Nordrhein-Westfalen) mit Zustimmung durch den Bundesdatenschutzbeauftragten eine Sondergenehmigung des Landesdatenschutzbeauftragten zur Recherche von Todesursachen bei verstorbenen Kohortenmitgliedern (auch ohne ihre vorhe- rige Einwilligungserklärung) beantragt werden. Für alle 671 Verstorbenen konnte das Sterbedatum - und somit die Überlebenszeit bzw. das erreichte Lebensalter - ermittelt werden. Für 539 Verstorbene $(80,45 \%)$ konnten zudem die Todesursachen recherchiert werden. Dabei waren in Übereinstimmung mit der amtlichen Todesursachenstatistik die meisten Todesfälle durch Herz-Kreislauf-Erkrankungen, gefolgt von Krebserkrankungen verursacht [17].

Der Ausfall von Nordrhein-Westfalen ist für das Mortalitäts-Follow-up als sehr problematisch anzusehen, da dadurch bei lange bestehenden bundesweiten Kohorten wie dem BGS98 und DEGS ein hoher Datenverlust für die Todesursachenrecherche zu verzeichnen ist. Zukünftig kann jedoch bei zeitnaher Recherche und durch Erfüllen der aktuellen Datenschutzbestimmungen (Einholen der Einwilligung der Studienteilnehmerinnen und -teilnehmer zur Todesursachenrecherche und in NRW das Einholen der zusätzlichen ärztlichen Schweigepflichtentbindung) eine nahezu vollständige Todesursachenrecherche durchgeführt werden. DEGS1-Probanten, die ab dem Sommer 2010 an dem Survey teilnahmen, wurden bereits um ihre Einwilligung gebeten und hatten in der Regel auch zugestimmt. Somit sind keine Akzeptanzprobleme zu erwarten. Datenverluste durch im Ausland Verstorbene und aufgrund der schwierigen Erhebungssituation in Berlin sind jedoch zu erwarten.

Das Erheben von Todesursachen ist unter den gegebenen Bedingungen mit hohem Zeit- und Personalaufwand verbunden. Auch muss damit gerechnet werden, dass einmal etablierte Abläufe fortlaufend angepasst werden müssen, da sich die länderspezifischen Rahmenbedingungen ändern können. Zudem gibt es in einigen Bundesländern (Berlin, NordrheinWestfalen, Rheinland-Pfalz) kurze Aufbewahrungsfristen für die Todesbescheinigungen und schwierige Zugangsbedingungen. Daher ist eine kontinuierliche Erhebung in regelmäßig wiederkehrenden Abständen von maximal 6 Monaten zwingend notwendig.

Ein international diskutierter Kritikpunkt bei der Erhebung von Todesursachen ist die eingeschränkte Validität der 
auf Leichenschauscheinen dokumentierten Daten [18-28]. Dies betrifft sowohl die Richtigkeit als auch die Vollständigkeit der Angaben. So ist der den Tod bescheinigende Arzt häufig nicht mit der Krankengeschichte des Verstorbenen vertraut. Hinzu kommen Zeitdruck, situative Zuständigkeiten, mangelndes Verständnis und Training der Ärzte sowie uneinheitliche Dokumentationsstandards (z. B. zwischen den Bundesländern variierende Gestaltung der Formulare). Die vorgenommenen Einträge sind nicht selten falsch platziert, unvollständig, unleserlich oder widersprüchlich. Maßnahmen zur Verbesserung der Datenqualität müssen daher sowohl Verbesserungen der Strukturals auch der Prozessqualität zum Ziel haben.

Die in der Literatur beschriebene Problematik der Validität der Todesbescheinigungen kann durch unsere Studie bestätigt werden. Da die Todesbescheinigungen aber so gestaltet sind, dass die Ärzte mehrere verschiedene Diagnosen eintragen sollen, ist eine Fehlerüberprüfung und Verbesserung der Datenqualität häufig möglich und im Interesse einer möglichst validen Todesursachenanalyse essenziell. Auch die Zusammenarbeit mit DIMDI bei der Überprüfung der Kodierung der Diagnosen und der Bestimmung des Grundleidens trägt erheblich zur Datenqualität bei, da hierdurch zentralisiert eine einheitliche, standardisierte Kontrolle und ggf. Umkodierung des zum Tode führenden Grundleidens nach Kriterien des international etablierten IRISSystems erfolgen kann.

Die dargestellten grundlegenden Ergebnisse der Überlebenszeitanalyse nach Alter und Geschlecht weisen auf die Anwendungsmöglichkeiten hin, die sich mit dem Mortalitäts-Follow-up für das Gesundheitsmonitoring und für epidemiologische Längsschnittanalysen bieten. Wie vor dem Hintergrund der amtlichen Statistik erwartet [17], findet sich auch unter den BGS98-Teilnehmenden eine geringere Überlebenswahrscheinlichkeit für Männer, sowohl in der altersgruppenspezifischen, als auch in der für Altersunterschiede kontrollierten Analyse. Derzeit schon laufende Auswertungsprojekte befassen sich mit zahlreichen vertiefenden Fragestellungen mit hoher Public-Health-
Relevanz, z. B. mit dem Einfluss des Blutzuckerstoffwechsels, der psychischen Gesundheit und des Gesundheitsverhaltens auf die Sterblichkeit in der Allgemeinbevölkerung in Deutschland.

Recherchen zur allgemeinen Mortalität und ursachenspezifischen Mortalität im Kontext von Kohortenstudien auf regionaler oder nationaler Ebene sind zur Überprüfung von Hypothesen zu kausalen Zusammenhängen zwischen Risikoexpositionen und gesundheitlichen Folgen essenziell und auch in Deutschland Bestandteil entsprechender Studien [2935]. Im Kontext des bundesweiten Gesundheitsmonitorings und der Weiterbeobachtung von Teilnehmenden bundesweiter Gesundheitssurveys stehen dagegen andere Zielsetzungen im Vordergrund. Von besonderer Bedeutung ist hierbei etwa die relative Mortalität von Erwachsenen mit Diabetes im Vergleich zu Nichtdiabetikern als Indikator der Versorgungsqualität bei Diabetes mellitus [36]. Der Nutzen der amtlichen Todesursachenstatistik ist hier limitiert, da Diabetes häufig nicht als Grundleiden gelistet wird. Ebenso bietet sich die Möglichkeit, anhand eines entsprechenden Vergleichs sozialer Gruppen die zeitliche Entwicklung sozialer Ungleichheit in Gesundheit und Sterblichkeit zu beobachten. Weiterhin eröffnet ein komplettes und kontinuierliches Mortalitäts-Follow-up von Teilnehmerinnen und Teilnehmern bundesweiter Gesundheitssurveys prognostische Bewertungen unterschiedlichster Kombinationen von modifizierbaren Risikofaktoren für chronische Krankheiten.

\section{Ausblick}

Das Mortalitäts-Follow-up der DEGSStudie hat den Grundstein für einen weiteren wichtigen Bestandteil des bundesweiten Gesundheitsmonitorings gelegt. Mit ihm eröffnet sich die Perspektive für Auswertungen zu Krankheitsverläufen, Krankheitsfolgen und prognostisch relevanten Risikokonstellationen im Hinblick auf den Endpunkt Sterblichkeit. Damit lassen sich Einschätzungen zu Versorgungsqualität und Präventionspotenzial bei Erwachsenen in Deutschland untermauern. Dies trägt dazu bei, eine Basis für eine sinnvolle und rationale Planung von
Prävention und Gesundheitsversorgung zu schaffen. Mit dem Mortalitäts-Followup konnten vollständige Daten zur allgemeinen Sterblichkeit erhoben werden. Eine Todesursachenrecherche war unter den gegebenen Rahmenbedingungen nur in $80 \%$ der Todesfälle möglich und mit hohem Aufwand verbunden. Eine regelmäßige und zeitnahe Recherche ist essenziell, um die Vollständigkeit der Informationen zu sichern. Dazu muss das Mortalitäts-Follow-up als fester Bestandteil des Gesundheitsmonitorings im RKI etabliert werden.

\section{Korrespondenzadresse}

\section{I.-K. Wolf MPH}

Robert Koch-Institut

Abt. 2, General-Pape-Straße 62-64/66

12101 Berlin

wolfi@rki.de

Danksagung. Wir bedanken uns beim Bundesdatenschutzbeauftragen sowie allen Landesdatenschutzbeauftragten und ihren Mitarbeitern aus dem Ressort Wissenschaft und Forschung für ihre Unterstützung. Unser Dank gilt den Gesundheitsministerien der Länder, dem Gemeinsamen Krebsregister Berlin-Brandenburg, dem Amt für Statistik Berlin, den mitwirkenden Ärzten Berlins, dem Deutschen Landkreistag, dem Deutschen Institut für Medizinische Dokumentation und Information (DIMDI), den Krankenhäusern und Standesämtern und allen Gesundheitsämtern, die uns bundesweit unterstützt haben. Auch bei allen Kolleginnen und Kollegen im RKI, die uns bei unterschiedlichen Fragen mit Rat und Tat unterstützt haben, möchten wir uns bedanken. Insbesondere gilt unser Dank allen früheren und zukünftigen Studienteilnehmerinnen und Teilnehmern, die mit ihrer Studienteilnahme entscheidend zum Wissen über die Gesundheit der Bevölkerung beitragen. Ohne ihre Hilfe wäre diese Studie nicht möglich gewesen.

Finanzierung. Das Mortalitäts-Follow-up der Studie zur Gesundheit Erwachsener in Deutschland (DEGS) wurde vom Bundesministerium für Gesundheit (BMG) finanziert.

\section{Einhaltung ethischer Richtlinien}

Interessenkonflikt. I.-K. Wolf, M. Busch, M. Lange, P. Kamtsiuris, R. Doelle, A. Richter, R. Kuhnert, T. Ziese, H. Knopf und C. Scheidt-Nave geben an, dass kein Interessenkonflikt besteht.

DEGS wurde mit Zustimmung der Ethikkommission der Charité Berlin und unter Berücksichtigung der Empfehlungen des Bundesbeauftragten für Datenschutz durchgeführt. Für das Mortalitäts-Follow-up wurden die Stellungnahmen des Bundesbeauftragten für Datenschutz und der Landesbeauftragten für Datenschutz eingeholt. 


\section{Literatur}

1. Robert Koch-Institut (2009) DEGS - Studie zur Gesundheit Erwachsener in Deutschland. Projektbeschreibung. In: Gesundheitsberichterstattung des Bundes. RKI, Berlin

2. Scheidt-Nave C, Kamtsiuris P, Gößwald A, Hölling $H$, Lange M, Busch MA et al (2012) German health interview and examination survey for adults (DEGS) - design, objectives and implementation of the first data collection wave. BMC Public Health $12: 730$

3. Gößwald A, Lange M, Kamtsiuris $P$, Kurth B-M (2012) DEGS: German Health Interview and Examination Survey for Adults. A nationwide cross-sectional and longitudinal study within the framework of health monitoring conducted by the Robert Koch Institute. Bundesgesundheitsbl Gesundheitsforsch Gesundheitsschutz 55:755-780

4. Kamptsiuris $P$, Lange $M$, Hoffmann $R$, Schaffrath Rossario A, Dahm S, Kuhnert R et al (2013) Die erste Welle der Studie zur Gesundheit Erwachsener in Deutschland (DEGS1). Stichprobendesign, Response, Gewichtung und Repräsentativität. Bundesgesundheitsbl Gesundheitsforsch Gesundheitsschutz 56:620-630

5. Bellach BM, Knopf H, Thefeld W (1998) The German Health Survey 1997/98. Gesundheitswesen 60:59-68

6. Stafford N (2010) Germany moves closer to establishing a national mortality register. BMJ 340:c2517

7. Giersiepen K, Brunings-Kuppe C et al (2004) The Bremen mortality index. Bundesgesumdheitsbl Gesundheitsforsch Gesundheitsschutz 47:451-456

8. Landesdatenschutzgesetze. http://www.bfdi. bund.de/DE/AnschriftenUndLinks/Landesdatenschutzbeauftragte/AnschriftenLandesdatenschutzbeauftragte.html. Zugegriffen: 10. Mai 2012

9. Bundesdatenschutzgesetz. http://www.bfd.bund. de/. Zugegriffen: 10. Mai 2012

10. Wolf I-K, Knopf H, Scheidt-Nave C, Kurth B-M (2012) Möglichkeiten und Grenzen retrospektiver Todesursachenrecherchen im Rahmen bundesweiter epidemiologischer Studien. Bundesgesundheitsbl Gesundheitsforsch Gesundheitsschutz 55:431-435

11. Metschke R, Wellbrock R (2004) Datenschutz in Wissenschaft und Forschung. Berliner Beauftragter für Datenschutz und Informationsfreiheit und Hessischer Datenschutzbeauftragter. http://www. unimuenchen.de/einrichtungen/orga_Imu/beauftragte/dschutz/regelungen/ds_wiss_und_fo.pdf. Zugegriffen: 20. Jan. 2011

12. Gößwald A, Lange M, Dölle R, Hölling H (2013) Die erste Welle der Studie zur Gesundheit Erwachsener in Deutschland (DEGS1). Gewinnung von Studienteilnehmenden, Durchführung der Feldarbeit und Qualitätsmanagement. Bundesgesundheitsbl Gesundheitsforsch Gesundheitsschutz 56:611-619

13. WHO (1979) Medical certification of cause of death. Instructions for physicians on use of international form of medical certification of cause of death. WHO, Geneva. http://whqlibdoc.who.int/publications/9241560622.pdf. Zugegriffen: 15. Juli 2014

14. European Commission (2013) Causes of death. Eurostat, European Commission 2013. http:// ec.europa.eu/health/major_chronic_diseases/ mortality/causes_death/index_en.htm. Zugegriffen: 15 . Juli 2014
15. DIMDI Medical Knowledge. German Institute of Medical Documentation and Information About Iris. http://www.dimdi.de/static/en/klassi/koop/ irisinstitute/about-iris/index.htm. Zugegriffen: 17. Juli 2014

16. DIMDI Medical Knowledge. German Institute of Medical Documentation and Information (2013) Iris News. http://www.dimdi.de/static/ en/klassi/koop/irisinstitute/news/news_0001. html_319159481.html.Zugegriffen: 8. Juli 2014

17. Robert Koch-Institut (Hrsg) (2011) Sterblichkeit, Todesursachen und regionale Unterschiede. Gesundheitsberichterstattung des Bundes. Heft 52. RKI, Berlin

18. Aung E, Rao C, Walker S (2010) Teaching cause-ofdeath certification: lessons from international experience. Postgrad Med J 86:143-152

19. Berry SD, Ngo L et al (2010) Competing risk of death: an important consideration in studies of older adults. J Am Geriatr Soc 58:783-787

20. Ermenc B (2000) Comparison of the clinical and post mortem diagnoses of the causes of death. Forensic Sci Int 114:117-119

21. Klug SJ, Bardehle D, Ressing M, Schmittmann I, Blettner M (2009) Vergleich von ICD-Kodierung zwischen Mortalitätsstatistik und studieninterner retrospektiver Nachkodierung. Gesundheitswesen 71:220-225

22. Madea B, Dettmeyer R (2003) Ärztliche Leichenschau und Todesbescheinigung. Kompetente Durchführung trotz unterschiedlicher Gesetzgebung der Länder. Dtsch Ärzteblatt 100:3161-3179

23. Maudsley G, Williams EMI (1996) "Inaccuracy" in death certification - where are we now? J Public Health Med18:59-66

24. Projekt Leichenschauscheine im Main-Kinzig-Kreis Hessen (2008) Überprüfung von Leichenschauscheinen hinsichtlich systematischer formaler und inhaltlich logischer Fehler von Januar 2008 bis Dezember 2008. Ein Projekt des Gesundheitsamtes Main-Kinzig-Kreis, Hessen

25. Schelhase T, Weber S (2008) Todesursachenverschlüsselung auf dem Totenschein. Fortbildung für den öffentlichen Gesundheitsdienst am 02.04.2008, Berlin. http://www.bfr.bund.de/ $\mathrm{cm} / 343$ /todesursachenverschluesselung_auf_ dem_totenschein_weber.pdf. Zugegriffen: 10. Dez. 2013

26. Alpérovitch $A$, Bertrand $M$, Jougla E, Vidal J, Ducimetière $P$, Helmer $C$ et al (2009) Do we really know the cause of death of the very old? Comparison between official mortality statistics and cohort study classification. Eur J Epidemiol 24:669-675

27. Johansson L, Westerling R, Rosenberg H (2006) Methodology of studies evaluating death certificate accuracy were flawed. J Clin Epidemiol 59:125131

28. Saydah S, Geiss L, Tierney E, Benjamin S, Engelgau $M$, Brancati F (2004) Review of the performance of methods to identify diabetes cases among vital statistics, administrative, and survey data. Ann Epidemiol 14:507-516

29. Helmert U (2003) Individuelle Risikofaktoren, Gesundheitsverhalten und Mortalitätsentwicklung in Deutschland im Zeitraum 1984 bis 1998. Gesundheitswesen 65:542-547

30. Klein T, Schneider S, Löwel H (2001) Bildung und Mortalität. Die Bedeutung gesundheitsrelevanter Aspekte des Lebensstils. Z Soziol 30:384-400
31. Herder C, Baumert J, Zierer A, Roden M, Meisinger C, Karakas M et al (2011) Immunological and cardiometabolic risk factors in the prediction of type 2 diabetes and coronary events: MONICA/KORA Augsburg Case-Cohort Study. PLOS ONE, June 06 2011. http://www.plosone.org/article/info\%3Adoi\%2F10.1371\%2Fjournal.pone.0019852. Zugegriffen: 20. Juni 2012

32. Wichmann HE, Kaaks R, Hoffmann W, Jöckel KH, Greiser KH, Linseisen J (2012) Die Nationale Kohorte. Bundesgesundheitsbl Gesundheitsforsch Gesundheitsschutz 55:781-789

33. Haerting J, Kluttig A, Greiser KH, Nuding S, Werdan K (2012) Kohortenstudie zu Risikofaktoren für Herz-Kreislauf-Krankheiten in einer urbanen älteren ostdeutschen Allgemeinbevölkerung (CARLAStudie). Bundesgesundheitsbl 6-7(55):795-800

34. Bergmann MM, Bussas U, Boeing H (1999) Follow-up procedures in EPIC-Germany - data quality aspects. European Prospective Investigation into Cancer and Nutrition. Ann Nutr Metab 43:225-234

35. Schwedhelm E, Wallaschofski H, Atzler D, Dörr M, Nauck M, Völker U et al (2014) Incidence of all-cause and cardiovascular mortality predicted by symmetric dimethylarginine in the population-based study of health in Pomerania. PLoS One 9. http://www.plosone.org/article/info\%3Adoi\%2F10.1371\%2Fjournal.pone.0096875. Zugegriffen: 1. Juli 2014

36. Gregg E, Cheng YJ, Saydah S, Cowie C, Garfield S, Geiss L et al (2012) Trends in death rates among U.S. adults with and without diabetes between 1997 and 2006: findings from the National Health Interview Survey. Diabetes Care 35(6):1252-1257 